\title{
Discovery of Novel Transcription Factor Inhibitors Using a Pyrazole-based Small Molecule Library
}

\author{
Hyung-Ho Ha and B. Moon Kim* \\ Deparment of Chemistry, Seoul National Lniversity, Seoul 151-747, Korea. ${ }^{\times}$E-mail kimbmasnu.ac.kr \\ Received October 18, 2007
}

\begin{abstract}
A focused library of pyrazole-based compounds was constructed towards novel transcription factor inhibitors. Complementary hydrogen bonding interaction with $\beta$-sheet peptide structures was the basis for the design of 5-amino-3-pyrazole carboxamide scaffold. From the preliminary inhibition assay against several transcription factors. compounds $7 \mathbf{e}$ and $\mathbf{8 g}$ were identified as novel lead compounds against $\mathrm{HF}-\mathrm{l} \alpha$ and NF-AT transcription factors. respectively:
\end{abstract}

Key Words : Transcription factor. 5-Amino-3-pyrazole carboxanude, HIF-1 $\alpha$ NF-AT, Inlibitor

\section{Introduction}

Transcription factors are a family of proteins that are involved in the regulatory control of various cellular events such as the gene expression and cytokine production. ${ }^{1}$ Many aspects of eukaryotic biology is hinged on the regulation of gene expression by transcription factors. ${ }^{23}$ Transcription factors that become overactive in most human cells are known to be important in cancer and inflammatory diseases. These proteins mediate excessive transcription of genes whose products are required for tumor growth and metastasis. ${ }^{4}$ Inhibiting the function of a transcription factor requires specific disnuption of DNA-protein or protein-protein interactions. ${ }^{5}$ Regulation of such interactions through the use of small organic molecules is a particularly important target of research. A number of attempts have been made to mimic transcription factors using sy'nthetic molecules. However the presence of peptide regions in these molecules may limit their future applications.

Recently Weluner and Schrader reported 3-aminopyrazole ${ }^{\mathrm{ll}}$ derivatives 1 as effective $\beta$-sheet ligands capable of stabilizing an extended conformation. Sy'nthetic polyamides containing $N$-methylimidazole and $N$-methylpyrrole amino acids targeted to a specific region of the transcription factor binding site have been reported." As shown in Figure 1. Nacylated 3-aminopyrazoles were shown to interact with every hỵdrogen-bond-donor (D) and acceptor (A) available
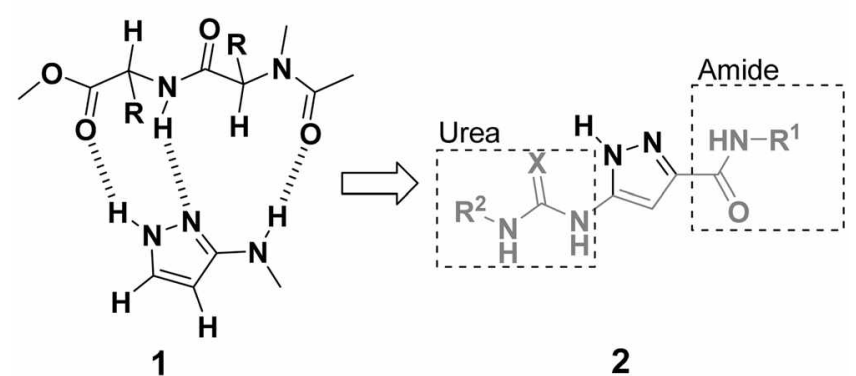

Figure 1. Peptidomimetic pyrazole amino acid from the DAD binding sites of the aminopvrazole. at the top face of a dipeptide. Based upon this intriguing feature of the aminopyrzole moiety. we envisioned that 5amino-3-pyrazole carboxamide derivative $\mathbf{2}$ with even further extended hydrogen bond capability would be extremely useful in binding certain extended peptide motifs. Herein we report on the design. sy'nthesis. and transcription factor inhibitory activities of a focused library of pyrazole amino acid derivatives.

\section{Results and Discussion}

Chemistry. A general synthesis of the pyrazole amino acid derivatives 2 is outlined in Scheme 1. All syntheses were carried out in a solution phase. Coupling of commercially available 5-nitro-3-pyrazolecarboxylic acid 3 with an appropriate amine in presence of HATU (2-(1H-7-azabenzotriazol-1-yl)-1.1.3.3-tetramethyluronium hexafluorophosphate) in $\mathrm{CH}_{2} \mathrm{Cl}_{2}$ provided pyrazole anide 4 . Reduction with $\mathrm{Na}_{2} \mathrm{~S}_{2} \mathrm{O}_{4}$ in presence of a phase transfer catalyst ${ }^{12}$ converted
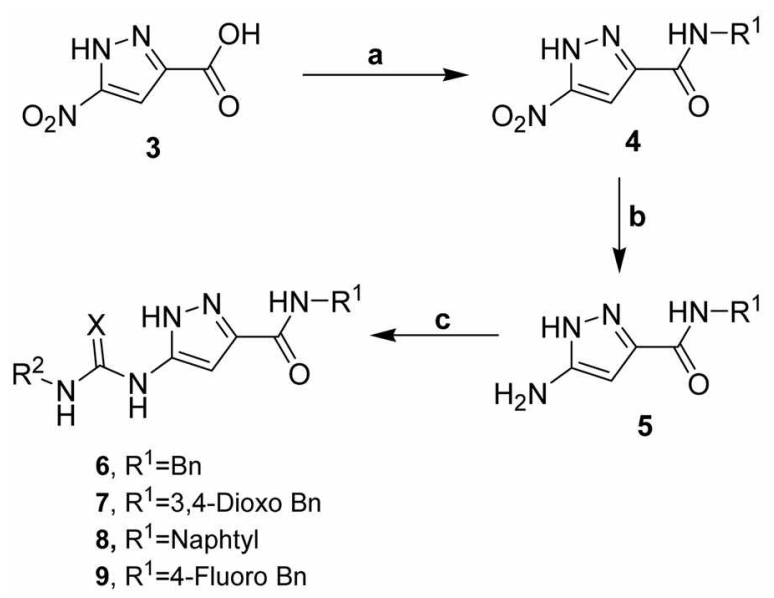

Scheme 1. A general synthetic scheme for pyrazole amino acid derivatives. Reagents and conditions: (a) HATU, " $\mathrm{Pr}_{2} \mathrm{NEt}_{3} \mathrm{CH}_{2} \mathrm{Cl}_{2}$, room temperature, $6 \mathrm{~h}$; (b) $\mathrm{Na}_{2} \mathrm{~S}_{2} \mathrm{O}_{4}, 0.5 \mathrm{~N}$ aq. $\mathrm{K}_{2} \mathrm{CO}_{3}, \mathrm{CH}_{2} \mathrm{Cl}_{2}$, cat. tetrabutylammonium bromide, room temperature, $6 \mathrm{~h}$ : (c) isocyanate, THF, room temperature, $8 \mathrm{~h}$. 
Table 1. Spectral data of pyrazole amino acid library<smiles>[R]NC(=O)c1cc(NC([X])N[R])[nH]n1</smiles>

\begin{tabular}{|c|c|c|c|c|c|}
\hline $\begin{array}{l}\text { Compd } \\
\text { No. }\end{array}$ & Code No. & $\mathrm{R}^{1}$ & $\mathrm{R}^{\hat{2}}$ & $\mathrm{X}$ & 'H NMR (300 MHz, DMSO-d $\mathrm{d}_{s} \delta$ ppm ) \\
\hline $6 a$ & $\mathrm{H}-\mathrm{I}-052$ & $\mathrm{Bn}$ & Pli & 0 & $\begin{array}{l}10.20(\mathrm{~s}, 1 \mathrm{H}), 8.85(\mathrm{t}, 1 \mathrm{H}), 7.70(\mathrm{~d}, 2 \mathrm{H}), 7.45(\mathrm{~d}, 2 \mathrm{H}), 7.30(\mathrm{~m}, 3 \mathrm{H}), 7.20(\mathrm{~m}, 3 \mathrm{H}) \\
6.65(\mathrm{~s}, 2 \mathrm{H}), 5.70(\mathrm{~s}, 1 \mathrm{H}), 4.50(\mathrm{~d}, 2 \mathrm{H})\end{array}$ \\
\hline $6 \mathrm{~b}$ & $\mathrm{H}-\mathrm{I}-0.53$ & $\mathrm{Bn}$ & $4-\mathrm{CH}_{3} \mathrm{OPh}$ & 0 & $\begin{array}{l}9.80(\mathrm{~s}, \mathrm{lH}), 8.80(\mathrm{t}, 1 \mathrm{H}), 7.55(\mathrm{~m}, 2 \mathrm{H}), 7.30(\mathrm{~m}, 2 \mathrm{H}), 7.20(\mathrm{~m}, 2 \mathrm{H}), 7.05(\mathrm{~m}, 3 \mathrm{H}), \\
6.60(\mathrm{~s}, 2 \mathrm{H}), 5.70(\mathrm{~s}, 1 \mathrm{H}), 4.50(\mathrm{~d}, 2 \mathrm{H}), 3.75(\mathrm{~s}, 3 \mathrm{H})\end{array}$ \\
\hline $7 a$ & $\mathrm{H}-\mathrm{I}-054$ & 3,4-diosol Bn & $4-\mathrm{CH}_{3} \mathrm{Ph}$ & 0 & $\begin{array}{l}10.25(\mathrm{~s}, 1 \mathrm{H}), 8.67(\mathrm{t}, 1 \mathrm{H}), 7.50(\mathrm{~d}, 2 \mathrm{H}), 7.35(\mathrm{~d}, 2 \mathrm{H}), 6.85(\mathrm{~m}, 3 \mathrm{H}), 6.63(\mathrm{~s}, 2 \mathrm{H}), \\
5.98(\mathrm{~s}, 2 \mathrm{H}), 5.65(\mathrm{~s}, 1 \mathrm{H}), 4.35(\mathrm{dd}, 2 \mathrm{H}), 2.30(\mathrm{~s}, 3 \mathrm{H})\end{array}$ \\
\hline $7 b$ & $\mathrm{H}-\mathrm{I}-055$ & 3,4-dioxol Bn & $4-\mathrm{CF}_{3} \mathrm{Plh}$ & 0 & $\begin{array}{l}10.26(\mathrm{~s}, 1 \mathrm{H}), 8.65(\mathrm{t}, 1 \mathrm{H}), 7.85(\mathrm{~d}, 2 \mathrm{H}), 7.75(\mathrm{~d}, 2 \mathrm{H}), 6.85(\mathrm{~m}, 3 \mathrm{H}), 6.65(\mathrm{~s}, 2 \mathrm{H}) \\
5.99(\mathrm{~s}, 2 \mathrm{H}), 5.71(\mathrm{~s}, 1 \mathrm{H}), 4.40(\mathrm{dd}, 2 \mathrm{H})\end{array}$ \\
\hline $7 c$ & H-I-056 & 3,4-dioxol Bn & 2-FPh & 0 & 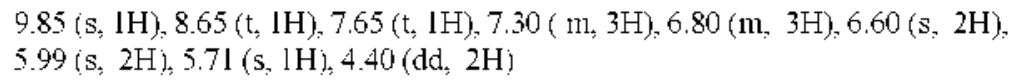 \\
\hline $7 d$ & H-I-057 & 3,4-dioxol Bn & $4-\mathrm{CH}_{2} \mathrm{OPh}$ & $\mathrm{O}$ & $\begin{array}{l}9.86(\mathrm{~s}, \mathrm{HH}), 8.65(\mathrm{t}, 1 \mathrm{H}), 7.50(\mathrm{~d}, 2 \mathrm{H}), 6.95(\mathrm{~d}, 2 \mathrm{H}), 6.85(\mathrm{~d}, 2 \mathrm{H}), 6.80(\mathrm{~d}, 1 \mathrm{H}) \\
6.55(\mathrm{~s}, 2 \mathrm{H}), 5.99(\mathrm{~s}, 2 \mathrm{H}), 5.67(\mathrm{~s}, 1 \mathrm{H}), 4.40(\mathrm{dd}, 2 \mathrm{H}), 3.75(\mathrm{~s}, 3 \mathrm{H})\end{array}$ \\
\hline $7 e$ & H-I-058 & 3,4-dioxol Bn & 1-Naphỵl & $S$ & $\begin{array}{l}13.40(\mathrm{br}, \mathrm{lH}), 10.95(\mathrm{br}, \mathrm{lH}), 9.20(\mathrm{~s}, 1 \mathrm{H}), 7.80(\mathrm{~m}, 4 \mathrm{H}), 7.60(\mathrm{~m}, 3 \mathrm{H}), 6.80(\mathrm{~m}, \\
4 \mathrm{H}), 6.05(\mathrm{~s}, 2 \mathrm{H}, 4.35(\mathrm{dd}, 2 \mathrm{H})\end{array}$ \\
\hline $7 \mathrm{f}$ & H-I-059 & 3,4-dioxol Bn & n-Pentyl & 0 & $\begin{array}{l}8.40(\mathrm{t}, \mathrm{lH}), 8.15(\mathrm{t}, \mathrm{lH}), 6.82(\mathrm{~m}, 3 \mathrm{H}), 6.47(\mathrm{~s}, 2 \mathrm{H}), 5.95(\mathrm{~s}, 2 \mathrm{H}), 5.65(\mathrm{~s}, 1 \mathrm{H}) \\
4.30(\mathrm{~d}, 2 \mathrm{H}), 3.20(\mathrm{~m}, 2 \mathrm{H}), 1.55(\mathrm{~m}, 2 \mathrm{H}), 1.30(\mathrm{~m}, 4 \mathrm{H}, 0.90(\mathrm{t}, 3 \mathrm{H})\end{array}$ \\
\hline $7 \mathbf{g}$ & $\mathrm{H}-\mathrm{I}-060$ & 3,4-diosol Bn & $n$-Butyl & 0 & $\begin{array}{l}8.42(\mathrm{t}, \mathrm{lH}), 8.13(\mathrm{t}, \mathrm{lH}), 6.80(\mathrm{~m}, 3 \mathrm{H}), 6.50(\mathrm{~s}, 2 \mathrm{H}), 5.97(\mathrm{~s}, 2 \mathrm{H}), 5.65(\mathrm{~s}, 1 \mathrm{H}) \\
4.30(\mathrm{~d}, 2 \mathrm{H}), 3.20(\mathrm{~m}, 2 \mathrm{H}), 1.55(\mathrm{~m}, 2 \mathrm{H}), 1.30(\mathrm{~m}, 2 \mathrm{H}), 0.90(\mathrm{t}, 3 \mathrm{H})\end{array}$ \\
\hline $7 \mathrm{~h}$ & $\mathrm{H}-\mathrm{I}-06 \mathrm{l}$ & 3.4-diosol Bn & Bn1 & 0 & $\begin{array}{l}8.70(\mathrm{t}, \mathrm{lH}), 8.45(\mathrm{t}, \mathrm{lH}), 7.30(\mathrm{~m}, 5 \mathrm{H}), 6.80(\mathrm{~m}, 2 \mathrm{H}), 6.50(\mathrm{~s}, 2 \mathrm{H}), 5.95(\mathrm{~s}, 2 \mathrm{H}), \\
5.65(\mathrm{~s}, 1 \mathrm{H}), 4.45(\mathrm{~d}, 2 \mathrm{H}), 4.30(\mathrm{~d}, 2 \mathrm{H})\end{array}$ \\
\hline $7 \mathbf{i}$ & H-I- 062 & Naphtyl & $4-\mathrm{CH}_{3} \mathrm{Ph}$ & 0 & $\begin{array}{l}9.85(\mathrm{~s}, \mathrm{lH}), 8.70(\mathrm{t}, \mathrm{lH}), 8.20(\mathrm{~d}, \mathrm{lH}), 7.90(\mathrm{~d}, 1 \mathrm{H}), 7.80(\mathrm{~m}, \mathrm{HH}), 7.55(\mathrm{~m}, 6 \mathrm{H}) \\
7.20(\mathrm{~d}, 2 \mathrm{H}), 6.60(\mathrm{~s}, 2 \mathrm{H}), 5.70(\mathrm{~s}, 1 \mathrm{H}), 500(\mathrm{~d}, 2 \mathrm{H}), 2.25(\mathrm{~s}, 3 \mathrm{H})\end{array}$ \\
\hline $8 a$ & $\mathrm{H}-\mathrm{I}-06 \hat{3}$ & Naphtyl & $4-\mathrm{CF}_{3} \mathrm{Ph}$ & 0 & $\begin{array}{l}10.25(\mathrm{~s}, \mathrm{lH}), 9.20(\mathrm{~d}, \mathrm{lH}), 8.70(\mathrm{t}, 1 \mathrm{H}), 8.20(\mathrm{~d}, \mathrm{lH}), 7.75(\mathrm{~m}, 9 \mathrm{H}), 6.70(\mathrm{~s}, 2 \mathrm{H}), \\
5.75(\mathrm{~s}, \mathrm{lH}), 5.00(\mathrm{~d}, 2 \mathrm{H})\end{array}$ \\
\hline $8 b$ & H-I-064 & Naphtyl & 2-FPh & 0 & $\begin{array}{l}9.80(\mathrm{~s}, \mathrm{lH}), 8.70(\mathrm{t}, \mathrm{lH}), 8.20(\mathrm{~d}, 1 \mathrm{H}), 7.95(\mathrm{~d}, 1 \mathrm{H}, 7.80(\mathrm{~m}, \mathrm{IH}), 7.55(\mathrm{~m}, 5 \mathrm{H}) \\
7.25(\mathrm{~m}, 3 \mathrm{H}), 6.60(\mathrm{~s}, 2 \mathrm{H}, 5.75(\mathrm{~s}, \mathrm{IH}, 4.95(\mathrm{~d}, 2 \mathrm{H})\end{array}$ \\
\hline $8 c$ & H-I-065 & Naphtyl & $4-\mathrm{CH}_{2} \mathrm{OPh}$ & 0 & $\begin{array}{l}9.80(\mathrm{~s}, 1 \mathrm{H}), 8.70(\mathrm{~m}, 1 \mathrm{H}), 8.30(\mathrm{~s}, 1 \mathrm{H}), 8.20(\mathrm{~d}, 1 \mathrm{H}), 7.90(\mathrm{~m}, 2 \mathrm{H}), 7.50(\mathrm{~m}, 4 \mathrm{H}), \\
6.80(\mathrm{~m}, 3 \mathrm{H}), 6.60(\mathrm{~s}, 2 \mathrm{H}), 5.75(\mathrm{~s}, 1 \mathrm{H}, 5.00(\mathrm{~d}, 2 \mathrm{H}), 3.75(\mathrm{~s}, 3 \mathrm{H})\end{array}$ \\
\hline $8 d$ & H-I-066 & Naphtyl & I-Naphyl & $S$ & $\begin{array}{l}13.50(\mathrm{~s}, \mathrm{H}), 11.55(\mathrm{~s}, \mathrm{lH}), 11.00(\mathrm{~s}, 1 \mathrm{H}), 9.25(\mathrm{~s}, 1 \mathrm{H}), 8.00(\mathrm{~m}, 7 \mathrm{H}), 7.55(\mathrm{~m}, \\
6 \mathrm{H}), 6.80(\mathrm{~s}, 1 \mathrm{H}, 5.00(\mathrm{~d}, 2 \mathrm{H})\end{array}$ \\
\hline $8 \mathrm{e}$ & H-I-067 & Naphtyl & n-Pentyl & $\mathrm{O}$ & $\begin{array}{l}8.50(\mathrm{t}, \mathrm{lH}), 8.20(\mathrm{~d}, 2 \mathrm{H}), 7.90(\mathrm{~m}, 2 \mathrm{H}), 7.50(\mathrm{~m}, 3 \mathrm{H}), 6.50(\mathrm{~s}, 2 \mathrm{H}), 5.70(\mathrm{~s}, \mathrm{lH}) \\
4.90(\mathrm{~d}, 2 \mathrm{H}), 3.20(\mathrm{~m}, 2 \mathrm{H}), 1.50(\mathrm{~m}, 2 \mathrm{H}), 1.10(\mathrm{~m}, 1 \mathrm{H}), 0.80(\mathrm{~m}, 3 \mathrm{H})\end{array}$ \\
\hline $8 f$ & H-I- -068 & Naphtyl & Allyl & 0 & $\begin{array}{l}8.55(\mathrm{t}, 1 \mathrm{H}), 8.30(\mathrm{t}, \mathrm{lH}), 8.20(\mathrm{t}, \mathrm{lH}), 7.90(\mathrm{~m}, 2 \mathrm{H}), 7.55(\mathrm{~m}, 4 \mathrm{H}), 6.50(\mathrm{~s}, 2 \mathrm{H}) \\
5.70(\mathrm{~s}, \mathrm{lH}), 5.20(\mathrm{~m}, 2 \mathrm{H}), 4.80(\mathrm{~d}, 2 \mathrm{H}), 3.80(\mathrm{~m}, 2 \mathrm{H})\end{array}$ \\
\hline $8 \mathrm{~g}$ & H-I- 669 & Naphtyl & n-Butyl & 0 & $\begin{array}{l}8.50(\mathrm{t}, \mathrm{HH}), 8.20(\mathrm{~m}, 2 \mathrm{H}), 7.90(\mathrm{~m}, 2 \mathrm{H}), 7.50(\mathrm{~m}, 4 \mathrm{H}), 6.50(\mathrm{~s}, 2 \mathrm{H}), 5.70(\mathrm{~s}, \mathrm{H}), \\
4.95(\mathrm{~d}, 2 \mathrm{H}), 3.20(\mathrm{~m}, 2 \mathrm{H}), 1.40(\mathrm{~m}, 4 \mathrm{H}), 0.85(\mathrm{~m}, 3 \mathrm{H})\end{array}$ \\
\hline $8 \mathrm{~h}$ & $\mathrm{H}-\mathrm{I}-070$ & Naphtyl & $4-\mathrm{ClPh}$ & 0 & $\begin{array}{l}10.10(\mathrm{~s}, 1 \mathrm{H}), 8.70(\mathrm{t}, 1 \mathrm{H}), 8.20(\mathrm{~d}, 1 \mathrm{H}), 7.90(\mathrm{~m}, 3 \mathrm{H}), 7.40(\mathrm{~m}, 12 \mathrm{H}), 6.60(\mathrm{~s}, 2 \mathrm{H}), \\
5.75(\mathrm{~s}, 1 \mathrm{H}), 5.00(\mathrm{~d}, 2 \mathrm{H})\end{array}$ \\
\hline $8 \mathrm{i}$ & $\mathrm{H}-\mathrm{I}-07 \mathrm{l}$ & 4-Fluoto Bn & $4-\mathrm{CF}_{3} \mathrm{Pl}$ & 0 & $\begin{array}{l}10.25(\mathrm{~s}, 1 \mathrm{H}), 8.80(\mathrm{t}, 1 \mathrm{H}), 7.80(\mathrm{~d}, 2 \mathrm{H}), 7.75(\mathrm{~d}, 2 \mathrm{H}), 7.30(\mathrm{~m}, 2 \mathrm{H}), 7.20(\mathrm{~m}, 2 \mathrm{H}), \\
6,70(\mathrm{~s}, 2 \mathrm{H}), 5.75(\mathrm{~s}, 1 \mathrm{H}), 4.50(\mathrm{~d}, 2 \mathrm{H})\end{array}$ \\
\hline $9 a$ & H-I-072 & 4-Fluoro Bn & 2-FPh & $\mathrm{O}$ & $\begin{array}{l}9.80(\mathrm{~s}, \mathrm{lH}), 8.75(\mathrm{t}, \mathrm{lH}), 7.70(\mathrm{t}, \mathrm{lH}), 7.35(\mathrm{~m}, 3 \mathrm{H}), 7.25(\mathrm{~m}, 2 \mathrm{H}), 7.10(\mathrm{~m}, 1 \mathrm{H}) \\
6.80(\mathrm{~m}, 1 \mathrm{H}), 6.70(\mathrm{~m}, 1 \mathrm{H}), 6.50(\mathrm{~m}, 1 \mathrm{H}), 5.75(\mathrm{~s}, 1 \mathrm{H}), 4.50(\mathrm{~d}, 2 \mathrm{H})\end{array}$ \\
\hline $9 b$ & $\mathrm{H}-\mathrm{I}-073$ & 4-Fluoro Bn & $4-\mathrm{CH}_{2} \mathrm{OPh}$ & 0 & $\begin{array}{l}9.80(\mathrm{~s}, 1 \mathrm{H}), 8.80(\mathrm{t}, 1 \mathrm{H}), 7.55(\mathrm{~m}, 2 \mathrm{H}), 7.30(\mathrm{~m}, 2 \mathrm{H}), 7.20(\mathrm{~m}, 2 \mathrm{H}), 7.05(\mathrm{~m}, 2 \mathrm{H}) \\
6.60(\mathrm{~s}, 2 \mathrm{H}), 5.70(\mathrm{~s}, 1 \mathrm{H}), 4.50(\mathrm{~d}, 2 \mathrm{H}), 3.75(\mathrm{~s}, 3 \mathrm{H})\end{array}$ \\
\hline $9 c$ & H-I-074 & 4-Fluoro Bn & I-Naphyl & S & $\begin{array}{l}13.50(\mathrm{~s}, \mathrm{lH}), 11.00(\mathrm{~s}, 1 \mathrm{H}, 9.30(\mathrm{~s}, \mathrm{lH}), 8.05(\mathrm{~m}, 4 \mathrm{H}), 7.60(\mathrm{~m}, 3 \mathrm{H}), 7.35(\mathrm{~m}, \\
2 \mathrm{H}), 7.25(\mathrm{~m}, 2 \mathrm{H}), 6.75(\mathrm{~s}, \mathrm{lH}), 4.50(\mathrm{~d}, 2 \mathrm{H})\end{array}$ \\
\hline $9 d$ & $\mathrm{H}-\mathrm{I}-075$ & 4-Fluoro Bn & n-Pentyl & 0 & $\begin{array}{l}8.70(\mathrm{t}, \mathrm{lH}), 8.20(\mathrm{t}, \mathrm{lH}), 7.30(\mathrm{~m}, 2 \mathrm{H}), 7.20(\mathrm{~m}, 2 \mathrm{H}), 6.50(\mathrm{~s}, 2 \mathrm{H}), 5.65(\mathrm{~s}, 1 \mathrm{H}) \\
4.45(\mathrm{~d}, 2 \mathrm{H}), 3.20(\mathrm{~m}, 2 \mathrm{H}), 1.60(\mathrm{~m}, 2 \mathrm{H}), 1.25(\mathrm{~m}, 4 \mathrm{H}), 0.90(\mathrm{~m}, 3 \mathrm{H})\end{array}$ \\
\hline $9 \mathrm{e}$ & $\mathrm{H}-\mathrm{I}-076$ & 4-Fluoro Bn & Allyl & 0 & $\begin{array}{l}8.70(\mathrm{t}, \mathrm{lH}), 8.30(\mathrm{t}, \mathrm{lH}), 7.30(\mathrm{~m}, 2 \mathrm{H}), 7.20(\mathrm{~m}, 2 \mathrm{H}), 6.50(\mathrm{~s}, 2 \mathrm{H}), 5.80(\mathrm{~m}, 1 \mathrm{H}), \\
5.65(\mathrm{~s}, 1 \mathrm{H}), 5.15(\mathrm{~m}, 2 \mathrm{H}), 4.40(\mathrm{~d}, 2 \mathrm{H}), 3.80(\mathrm{~m}, 2 \mathrm{H})\end{array}$ \\
\hline $9 f$ & $\mathrm{H}-\mathrm{I}-0.077$ & 4-Fluoro Bn & n-Butyl & 0 & $\begin{array}{l}8.72(\mathrm{t}, \mathrm{lH}), 8.33(\mathrm{t}, \mathrm{lH}), 7.35(\mathrm{~m}, 2 \mathrm{H}), 7.25(\mathrm{~m}, 2 \mathrm{H}), 6.50(\mathrm{~s}, 2 \mathrm{H}), 5.60(\mathrm{~s}, 1 \mathrm{H}) \\
4.45(\mathrm{~d}, 2 \mathrm{H}), 3.25(\mathrm{~m}, 2 \mathrm{H}), 1.55(\mathrm{~m}, 2 \mathrm{H}), 1.30(\mathrm{~m}, 2 \mathrm{H}), 0.90(\mathrm{~m}, 3 \mathrm{H})\end{array}$ \\
\hline $9 \mathrm{~g}$ & H-I- -078 & 4-Fluoro Bn & 4-ClPh & 0 & $\begin{array}{l}10.10(\mathrm{~s}, \mathrm{HH}), 8.75(\mathrm{t}, 1 \mathrm{H}), 7.70(\mathrm{~d}, 2 \mathrm{H}), 7.45(\mathrm{~d}, 2 \mathrm{H}), 7.30(\mathrm{~m}, 2 \mathrm{H}), 7.20(\mathrm{~m}, 2 \mathrm{H}), \\
6.65(\mathrm{~s}, 2 \mathrm{H}), 5.70(\mathrm{~s}, \mathrm{H}), 4.50(\mathrm{~d}, 2 \mathrm{H})\end{array}$ \\
\hline
\end{tabular}



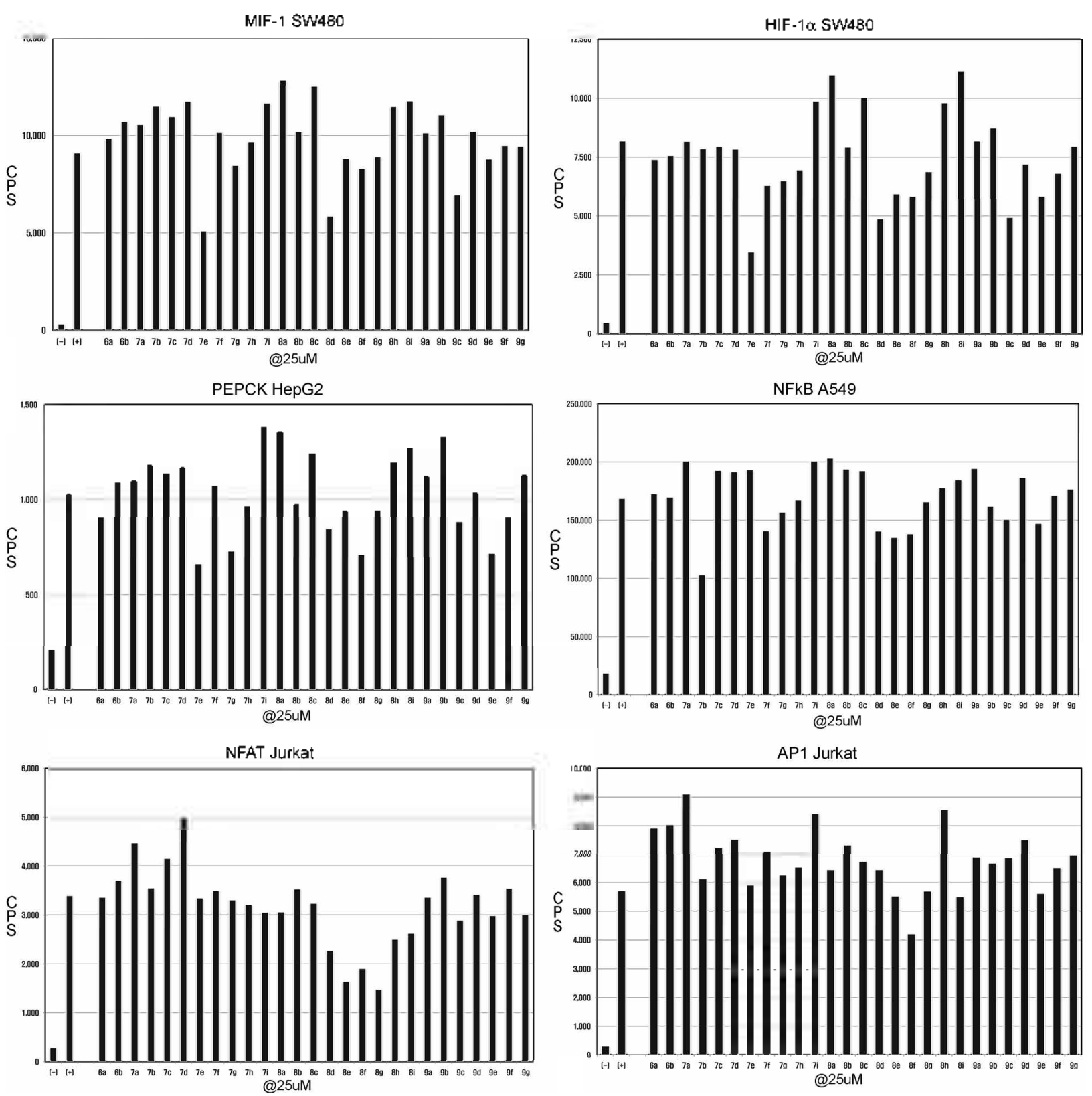

Figure 2. Results of the high throughput screens.

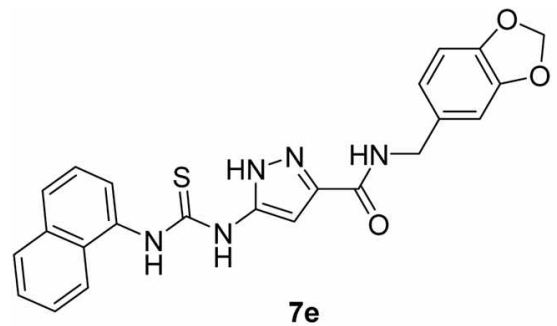

$61.1 \%$ inhibition against HIF-1 $\alpha$ in SW480 cell

Figure 3. Hit compounds against HIF-l $\alpha$ and NF-AT.

the nitro group of the amide 4 to an amine (compound 5 ). Subsequent coupling of $\mathbf{5}$ with an appropriate isocyanate or isothiocyanate gave the targeted structure 2 (compounds $6-$ 9). All compounds were purified through columu chromato-

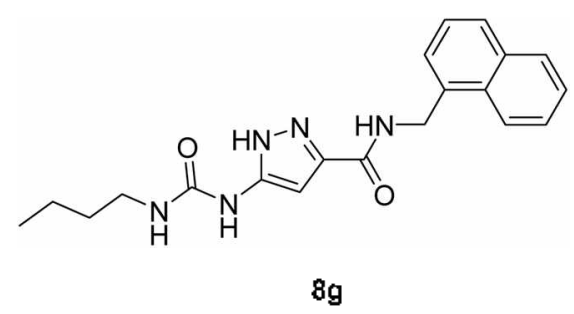

$61.4 \%$ inhibition against NF-AT in Jurkat cell

graphy to afford pure products. In Table 1, spectral data of each product are summarized.

High Throughput Screens. Herein, we report a preliminary screening result and identification of a series of novel 
inhibitors of transcription factors (MIF-1, HIF-1. PEPCK. NF-kB. NF-AT, and AP-1). Regulation of these transcription factors could lead to useful drug discovery targets in cancer and inflanmmatory: MIF-I for rheunatoid arthritis and atherosclerosis. ${ }^{13}$ HIF-l for cancer. heart disease and stroke. ${ }^{1+}$ PEPCK type 2 diabetes, ${ }^{15} \mathrm{NF}-\mathrm{kB}$ for asthma ${ }^{16} \mathrm{NF}-\mathrm{AT}$ for inflammatory bowel disease, psoriasis and multiple sclerosis, ${ }^{17}$ and AP. 1 for rheumatoid artluritis. ${ }^{18}$

The automated high-throughput assays were carried out with stably-transfected human cells (SW+80, HepG2. A549. and Jurkat) measuring luminescence from luciferase reporter gene assays. The screening results of the pyrazole library at $25 \mu \mathrm{M}$ concentration from the cell-based assays are summarized in Figure 2. As can be seen in Figure 2. the assays identified two compounds that selectively inhibited HIF-I $\alpha$ and NF-AT transcription factors. respectively. The structures of these compounds are depicted in Figure 3 .

In summary, we have discovered novel lead compounds that are effective transcription factor inhibitors from high throughput screening of a focused library of py razole anino acid derivatives. Among these compounds, $7 \mathrm{e}$ and $8 \mathrm{~g}$ showed the highest activity against HIF- $L$ and NF-AT. respectively.

\section{Experimental}

Starting materials were obtained from conmercial sources and used without purification. Silica gel (E. Merck, 60-230 mesh) was used for flash columun chromatography, and silica gel plates (E. Merck) were used for thin-layer chromatography. All NMR spectra were recorded on a Bruker DPX$300(300 \mathrm{MHz})$ and shifts are reported in parts per million relative to internal tetramethylsilane.

A general procedure for the coupling of an amine with carboxylic acid (4). To a solution of 5 -nitro- $1 H$-pyrazole-3carboxylic acid ( $314 \mathrm{mg}$. $2 \mathrm{mmol}$ ), 2-(1H-7-azabenzotriazol1-yl)-1,1.3.3-tetramethyluronium hexafluorophosphate (HATU) (912 mg. $2.4 \mathrm{mmol}$ ). DIPEA (0.83 mL. $4.8 \mathrm{mmol})$ in dichloromethane was added an appropriate amine (2 mmol). The reaction mixture was stirred for $6 \mathrm{~h}$ at room temperature. After the reaction was complete. the mixture was concentrated in vacto. The crude product was purified from silica gel chromatography:

A general procedure for the reduction of nitro group with $\mathrm{Na}_{2} \mathrm{~S}_{2} \mathrm{O}_{4}$ (5). To a solution of 5-nitro- $H$-pyrazole-3carboxylic amide (2 mmol), $\mathrm{Na}_{2} \mathrm{~S}_{2} \mathrm{O}_{4}(3$ eq. $6 \mathrm{mmol})$ in dichloromethane $(10 \mathrm{~mL})$ and $0.5 \mathrm{~N}$ aq. $\mathrm{K}_{2} \mathrm{CO}_{3}(5 \mathrm{~mL})$ was added cat. tetrabutylanmmonium bromide. The reaction mixture was stirred for $6 \mathrm{~h}$ at room temperature. The mixture was partitioned between dichloromethane and brine. Then the aqueous layer was extracted with dichloromethane (3 times). Combined organic layer was dried over anhy'drous sodium sulfate and solvent was removed under reduced pressure. The reaction mixture was concentrated in vacuo. The crude product was purified from silica gel chromatography:

General procedure for the coupling of isocyanate with amine (compounds 6-9). To a solution of 5-amino- $1 \mathrm{H}$ pyrazole-3-carboxylic amide ( $1 \mathrm{mmol}$ ) in THF was added appropriate isocyanate or isothiocyanate $(1.1 \mathrm{mmol})$. The reaction mixture was stirred for $6 \mathrm{~h}$ at room temperature. The reaction mixture was concentrated in vacuo. The crude product was purified from silica gel colunu cluromatography

General reporter-gene assay procedure using 96-well plate. I. Cells $(80 \mu \mathrm{L})$ were plated and cultured in white opaque 96-1vell tissue culture plates. Typical culture medium contained DMEM. 10\% fetal bovine serum and antibiotics (penicillin/streptonycin, gentanycin, etc). Amino acids and other nutrients were added to the culture medium. Assays were performed on either adherent cells or cells in suspension. The cells were either stably or transiently transfected with the luciferase gene. Culture volume of $80 \mu \mathrm{L}$ was used in this protocol. Blank control wells containing no cells were also prepared.

2. Test compounds and controls were added to cells. These were mixed well and incubated for the cells' desired period of time. Incubation time for gene regulation studies can be from several hours up to 3 days. Assays were run in duplicate or triplicate. A volume of $20 \mu \mathrm{L}$ compounds in PBS or culture medium was used.

3. The reagent was reconstituted. First the reagent and assay buffer were equilibrated to room temperature. Then the assay buffer and reagent were combined by pipetting a small volume (e.g. $1 \mathrm{~mL}$ ) buffer to the reagent tube. The mixture was vortexed briefly and the reconstituted solution was pipetted to the assay buffer bottle. This step was repeated to transfer all reagents to the assay buffer bottle. It was mixed by inversion until the reagent thoroughly dissolved. After this was done, the bottle label was marked as reconstituted reagent.

4. The reconstituted reagent ( $100 \mu \mathrm{L}$, per $80 \mu \mathrm{L}$ of cell culture) was added to each well and mixed well with the cells. The mixture was incubated for 2 minutes at room temperature. The volume of the reagent was adjusted depending on the volume of cell culture

5. Luminescence was measured on a luminometer. Reading was done with firefly substrate in dual luciferase assay șystem (promega).

Acknowledgements. We are grateful to Choongwae Pharmaceuticals for rumning the high throughput screens of transcription factors.

\section{References}

1. Manning. A. M. Drug Discoven Todav 1996. 1(4), 151-160.

2. Gottesfeld, J. M.: Neely. L.: Trauger, J. W: Baird, E. E.: Dervan. P. B. Natwe 1997. 387(6629), 202-205.

3. Denison. C.: Kodadek. T. Chentistry \& Biolog 1998. 5(6). Rl29RI45.

4. Darnell. I. E. Nature Review's Cancer 2002. 2(10). 740-749.

5. Tjian, R.: Maniatis. T. Cell 1994, 77(1), 5-8.

6. Mapp. A. K : Ansari. A. Z.; Ptashne, M.; Dervan, P. B. Proc. Nat. Acad Sci. 2000. 97(8), 3930-3935.

7. Ansari. A. Z.: Mapp. A. K.: Nguyen. D. H.: Derwan. P. B.: 
Ptashne. M. Chentisty \& Biolog 2001. \&(6). 583-592

8. Arora. P. S. Chentistiv \& Biologr 2005. I2(3). 262-263

9. Liu. B.: Han. Y: Corey. D. R.: Kodadek. T. J. Am. Chem. Soc. 2002, $127(9) .1838-1839$.

10. Djuric. S. W.: BaMaung. N. Y: Basha, A.: Liu. H.: Luly. I. R.: Madar. D. J.; Sciotti, R. I.: Tu. N. P: Wagenaar. F. L.: Wiedeman. P. E.: Zhou. X.: Ballaron. S.: Bauch. J.: Chen. Y.-W.: Chiou. X. G.: Fey. T.: Gauvin. D.: Gubbins. E.: Hsieh. G. C.: Marsh. K. C.: Mollison. K. W.: Pong. M.: Shaughnessy. T. K.: Sheets. M. P.: Smith, M.: Trevillyan. I. M.: Warrior, U.: Wegner. C. D.: Carter. G. W. J. Wed Chem. 2000. 43, $2975-2981$

11. Wehner, M.: Schrader. T. Angew: Chem. Int. Ed. 2002. H(10). $1751-1754$
12. Scheuerman. R. A.: Tumelty. D. Tetrahedron Lett. 2000. H1(34). $6531-6535$

13. Morand. E. F.: Leech. M.: Turgen. B. Nature Revien's Dntg Discovery 2006. $5(5), 399-410$.

14. Giaccia, A.: Sim, B. G.: Johnson, R. S. Natwe Reviews Dnug Discovery 2003. 2(10), 803-811.

15. Lazar. D. F.: Saltiel. A. R. Nature Review's Dhig Disconen 2006. $5(4) .333-342$

16. Barnes. P. J. Nature Review's Drug Disconery 2004. 3(10). $831-844$

17. O'Neill. L. A. J. Kanure Review's Dnug Discovery 2006. 5(7), 549563.

18. Smolen. J. S.: Steiner. G. Katwe Review's Drug Discoveny 2003. $2(6) .473-488$ 